\title{
Las políticas de libre elección de centro escolar como agentes causantes de desigualdad. Una Revisión Sistematizada de la Literatura
}

\author{
González López, Víctor \\ Facultad de Educación- Universidad de Salamanca, Salamanca, España \\ vgl@usal.es \\ Revesado Carballares, David \\ Facultad de Educación- Universidad de Salamanca, Salamanca, España \\ drevesado@usal.es \\ García Redondo, Eva \\ Facultad de Educación- Universidad de Salamanca, Salamanca, España \\ evagr@usal.es
}

\section{Resumen}

La globalización ha penetrado en la dinámica educativa mundial afectando a la política educativa de los diferentes estados. El avance del neoliberalismo ha traído arraigado un fuerte componente de libertad de los mercados, que al igual que a otras esferas, también ha afectado a la educación. Estas concepciones neoliberales de la globalización abogan principalmente por medidas que persiguen abrir la educación a los mercados, a la rendición de cuentas y a la descentralización. Entre todas estas destacan las políticas de libertad de elección de centro por parte de los padres, pero, ¿la libertad de elección de centro es generadora de desigualdades? En esta investigación, a través de una Revisión Sistemática de la Literatura de los últimos ocho años, se estudian los diferentes aspectos que pueden o no responder a la pregunta anteriormente planteada, desentrañando, para el caso español, los resultados de estas políticas de elección en desarrollo de procesos de igualdad o desigualdad entre alumnos.

\section{Abstract}

Globalization has broke through of the global educational dynamic affecting the educational policy of the different states. The advance of neoliberalism has brought with it a strong component of market freedom, which, like other spheres, has also affected education. These neoliberal conceptions of globalization mainly advocate actions that seek to open up education to markets, to accountability and to decentralization. Among all these actions, the policies of freedom of choice of the center by parents stand out, but is the freedom of choice of the center generating inequalities? In this research, through a Systematic Literature Review of the last eight years, the different aspects that may or may not respond to the above question will be studied, unraveling, for the Spanish case, the results of these election policies in development of equality or inequality processes among students.

Palabras clave: Desigualdad, Revisión Sistemática de la Literatura, Libertad de elección de centro, Política Educativa, Globalización.

Keywords: Inequality, Systematic Literature Review, Freedom of School Choice, Educational Policy, Globalization.

\section{INTRODUCCIÓN.}

La libertad educativa ha trazado, con mayor o menor éxito, la línea divisoria entre la libertad individual de los padres, los alumnos o los propios docentes y la libertad colectiva de todo un grupo. Una libertad educativa que está conformada por diferentes libertades o sub-libertades: libertad de creación y gestión de centro, libertad de elección de centro o educación por parte de los padres, libertad religiosa, libertad de cátedra o libertad de pensamiento del alumnado. Este caso que nos ocupa focalizará su intervención sobre la libertad de elección de centro, pero más concretamente como generadora de desigualdades sociales.

http://doi.org/10.25145/c.educomp.2018.16.063 
Hemos organizado esta intervención en base a las propiedades y principios de la Revisión Sistematizada de la Literatura en una primera aproximación a esta técnica de revisión bibliográfica caracterizada, principalmente, por su robustez y rigurosidad. Ligada a las Ciencias de la Salud nació una nueva metodología de análisis bibliográfico en contrapartida a las ineficaces revisiones subjetivas que no podían dar salida a todo el nuevo material académico que fluía en abundancia. Las revisiones sistemáticas nacieron, como expone Hunt, "como una metodología objetiva y rigurosa para llevar a cabo el proceso de revisión de la investigación en un campo concreto de conocimiento y lograr de esta forma una eficiente acumulación de las evidencias» (citado en Sánchez-Meca, 2010, p. 54). Ahora bien, existen dos formas de diferenciar o denominar a esta nueva metodología. De un lado, aquellos que utilizan la denominación sistemática, reservada para estudios pertinentes en el campo de las Ciencias de la Salud, y del otro, el término sistematizado, identificado con otros ámbitos como las Humanidades y las Ciencias Sociales donde los estudios cualitativos adquieren mayor preponderancia. Por lo tanto,

una revisión sistemática es un tipo de investigación [...] mediante la cual se revisa la literatura científica sobre un tópico partiendo de una pregunta formulada de forma clara y objetiva, utilizando métodos sistemáticos y explícitos para localizar, seleccionar y valorar críticamente las investigaciones relevantes a dicha pregunta y aplicando protocolos sistemáticos para la recogida de datos e información de dichas investigaciones, con el objetivo de alcanzar conclusiones válidas y objetivas sobre qué es lo que dicen las evidencias sobre dicho tópico (Sánchez-Meca, 2010, p. 54).

En definitiva, las revisiones sistemáticas pretenden sintetizar toda la información relevante sobre un determinado tema o tópico al margen de cualquier sesgo; "el término «sistemático» los distingue de los exámenes realizados sin métodos claros y responsables» (Gough, Thomas y Oliver, 2012, p. 2). Por lo tanto, «la metodología SLR ${ }^{1}$ tiene como objetivo sintetizar la evidencia científica existente en un área específica de conocimiento para responder a una o más preguntas de investigación» (Verger, Fontdevila y Zancajo, 2017, p. 25). Este tipo de revisión pasa por una serie de fases representadas en la figura 1, y que responden, de forma particular, a las seguidas en cualquier investigación empírica. Como recogen Verger et al (2017) y Sánchez-Meca (2010), en primer lugar, el investigador debe de plantear el problema o la pregunta de investigación que ha dado origen al estudio que presenta, de tal forma que ha identificado las necesidades, principalmente aquellas que sirven de motor para dar comienzo a la revisión sistematizada. En segundo lugar, ante la rigurosidad que caracteriza a este tipo de revisión, es necesario definir con claridad los criterios de búsqueda, delimitando las palabras clave que permitirán la selección de estudios. Seguidamente, se procederá a la extracción de datos relevantes obtenidos de las investigaciones seleccionadas según los criterios de búsqueda seleccionados con anterioridad para el estudio. El cuarto paso responde a la evaluación de la calidad y relevancia de los estudios y datos seleccionados. Por último, las etapas quinta y sexta precisan de la síntesis de los datos obtenidos y de su posterior difusión.

Imagen 1. Etapas comunes en la revisión sistemática.

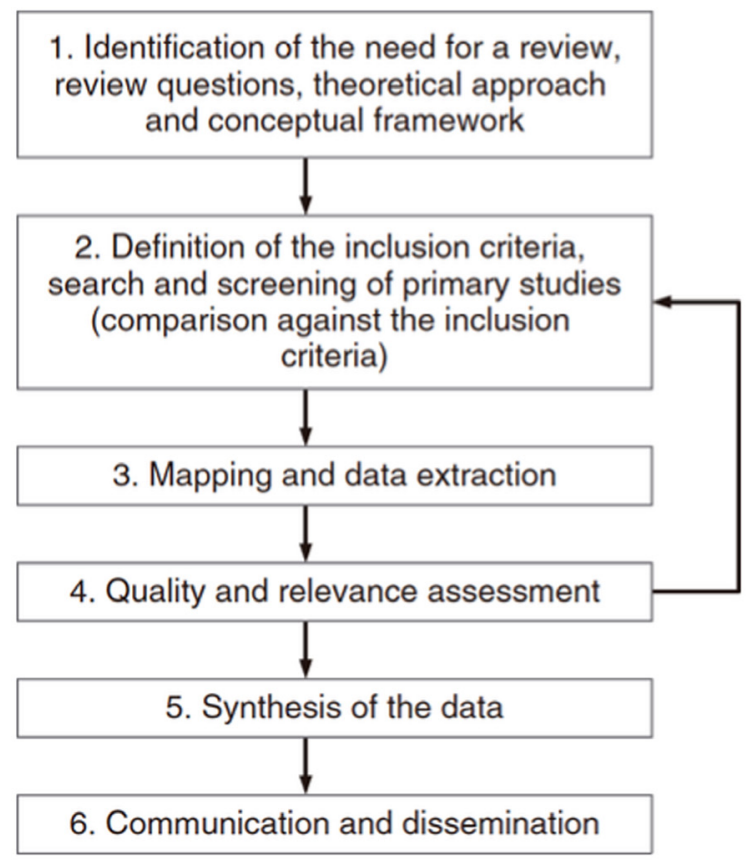

Fuente: Verger, Fondevila y Zancajo (2017, p. 10).

\footnotetext{
${ }^{1}$ Sistematic Literature Review (Análisis Sistemático de la Literatura).
} 


\section{METODOLOGÍA DE APLICACIÓN}

Para comenzar, nos hacemos la siguiente pregunta: ¿La libertad de elección de centro escolar genera igualdad de oportunidades o, por el contrario, es más propensa a perpetuar las desigualdades educativas? Partiendo de esta cuestión formulamos una hipótesis que afirma que «las políticas de libertad de elección de centro son causantes o generadoras de desigualdades sociales y educativas».

Para esta revisión se han seleccionado unos criterios de inclusión centrados diferentes factores:

- Artículos publicados en revistas académicas indexadas en las bases de datos SCOPUS y WOS.

- Artículos publicados en inglés y castellano

- Artículos referentes a Europa, Latinoamérica y Norteamérica.

- Publicaciones a partir del año 2010.

— Las palabras clave seleccionadas en la búsqueda que se encuentran en el resumen del artículo.

Los criterios de búsqueda han sido seleccionados según idioma, castellano e inglés, y según palabras clave. Para SCOPUS:

a. Libertad de elección.

b. Freedom of school choice.

c. Inequality freedom school choice.

Por su parte, los criterios de búsqueda seleccionados en WOS, al igual que en SCOPUS fueron:

a. Libertad de elección.

b. Freedom of school choice.

c. Inequality freedom school choice.

Tras estas búsquedas se han rescatado un total de 30 artículos entre las bases de WOS y SCOPUS que hagan referencia a los criterios de inclusión seleccionados. Tras esta recopilación, seleccionamos un total de 14 artículos, considerando su evaluación exhaustiva en función de la relevancia en la revisión. Por supuesto que entendemos que los criterios podrían haber sido mucho más amplios, pero esta trabajo se ha planteado como un primer acercamiento al tema de estudio, de tal forma que nos sirva como un incipiente espacio de conexión y se puedan arrojar una serie de conclusiones, con cierta relevancia, sobre el tema de estudio.

\section{RESULTADOS}

Tomando como punto de partida la hipótesis formulada — «las políticas de libertad de elección de centro son causantes o generadoras de desigualdades sociales y educativas»- con anterioridad a partir de nuestra pregunta de investigación, ofreceremos una revisión de la literatura obtenida siguiendo el proceso metodológico manifestado en el punto anterior.

En las últimas décadas, de la mano del progreso de la educación privada, en diferentes naciones se ha instaurado un nuevo modelo de gestión, donde conceptos provenientes del terreno económico han ganado especial relevancia, como la rendición de cuentas, la eficiencia y la eficacia y la propia privatización (Villalobos y Quaresma, 2015). Como señaló Olssen (citado por Mazzoli et al, 2017) las características del mercado neoliberal en el contexto educativo no hacen otra cosa que transformar a las personas en un producto. Estas nuevas condiciones han generado en muchas de las democracias liberales occidentales, políticas educativas basadas en un cuasi- mercado de la educación, que busca para sus clientes, padres y estudiantes, la libertad de elección, principalmente, de centro educativo (Savage, 2012), y que, así mismo, han dado lugar a un abierto debate político y científico.

Para Mazzoli, Todd y Laing (2017), en el privilegio en la elección de centro se observa un claro cariz neo- 
liberal, donde la promoción del derecho y libertad de elección de los padres, en función de las ofertas del mercado educativo, beneficia a los más favorecidos y con más opciones para elegir. Pero esto no es algo nuevo; ya en la década de los 60 del siglo pasado, cuando la escuela de masas había alcanzado sus cotas de participación más altas, algunos sectores poblacionales favorecidos social y económicamente decidieron costear de su bolsillo una educación ajena a la distribuida por el Estado (Narodowski, Gottau y Moschetti, 2016), dando lugar a unos nuevos procesos de selección, en este caso, basados en el privilegio. Posteriormente, unos veinte años después, en la década de los 80 , se ponen en marcha en Europa numerosas reformas educativas con el objetivo de desarmar los sistemas de gobierno centralizados en favor de nuevas estructuras descentralizadas, enfatizando con ello, en el terreno educativo, los mecanismos de elección de los padres, la libertad educativa o la competencia entre centros (Varjo y Kalalahti, 2014). Con todo ello, la elección de escuela ha pasado a ser una de las reformas educativas más extendidas, especialmente en la vertiente anglosajona (Tabatadze y Gorgadze, 2017). Estamos entonces, como señala Gubbins (2014) ante un nuevo modelo de distribución escolar, denominado «school choice, que se apoya en la convicción de que la eficacia escolar depende centralmente de los mecanismos y reglas del mercado aplicados al sistema de educación formal.» (p. 1070), y que, como reforma educativa, se está convirtiendo en una de las más mediatizadas.

Para Levin, Cornelisz y Hanisch-Cerda (2013, p. 516),

la libertad de elección es la característica dominante de un sistema educativo diseñado para capturar beneficios privados. Las familias elegirán las escuelas de acuerdo con sus valores, filosofías educativas, enseñanzas religiosas y tendencias políticas, así como la efectividad percibida en los resultados del aprendizaje. Los beneficios privados de la educación pueden ser maximizados por las familias si eligen escuelas que satisfagan plenamente sus aspiraciones educativas para sus hijos.

Esto ha dado lugar a dos importantes focos de debate relacionados con la elección libre de escuela y la equidad social; de un lado, aquellos que defienden un acceso igualitario a la educación, supervisado por el Estado, limitando la libertad de elección de los padres, en pos de la igualdad de oportunidades, la equidad educativa y la justicia social, y del otro, aquellos que defienden la privatización de la educación orientada hacia un modelo de mercado competitivo que supondría un incremento en la oferta educativa permitiendo a los padres elegir entre centros fuera de su radio de residencia (Levin, et al, 2013; Brando, 2017). Pero también, la libertad de elección ha sido interpretada en términos de justicia educativa, ofreciendo oportunidades a los niños más cualificados o con más talento, independientemente de su contexto social y económico (Varjo y Kalalahti, 2014). En definitiva, como señala Burnar (2010) se produce una confrontación de clases, un estrategia llevada a cabo por las familias de clase media y alta para asegurar su posición social y posterior reproducción cultural, alejando de su órbita a los colectivos no deseados. La igualdad de oportunidades ha sido entendida en la literatura desde cuatro enfoques diferenciados: la distribución equitativa de los recursos educativos, la igualdad de oportunidades en función de «outcomes» igualitarios, la igualdad entre iguales y la distribución de recursos en función de las necesidades individuales (Tabatadze y Gorgadze, 2017). El primero aboga por la distribución de los recursos de forma igualitaria para todos, de tal forma que cada ciudadano tenga los mismos derechos; el segundo se centra, específicamente, en la igualdad de oportunidades educativas en función de los resultados obtenidos por cada uno (meritocracia); el tercero sugiere que todos los niños con el mismo nivel de capacidad y disposición para aprender deberían tener la misma oportunidades de éxito independientemente de sus condiciones socioeconómicas; y el cuarto, defiende una distribución basada en la equidad, donde a cada individuo le correspondería una distribución de recursos en función de sus necesidades.

Tradicionalmente, las familias han accedido al puesto escolar primando la situación del hogar de residencia, es decir, mediante una distribución territorial donde el Estado o la autoridad pública correspondiente asigna a cada niño un centro escolar dentro del área donde se encuentra su vivienda habitual. En algunos países, por razones educativas y de elección de escuela esto ha dado lugar al crecimiento de los mercados de vivienda para acceder a las escuelas preferidas por los padres (Varjo y Kalalahti, 2014), es decir, las familias cambian su residencia en función del espacio geográfico donde se encuentre ubicado el centro escolar en el que quieren matricular a sus hijos. Pero este modelo de elección también produce desigualdad. La segregación social entre las escuelas debe considerarse como una consecuencia de la segregación residencial (Bartholo, 2013), es decir, las zonas de asistencia escolar, a menudo están definidas por barrios circunscritos a la clase social y raza (Levin et al, 2013) y donde muchas escuelas acaban convirtiéndose en guetos alejados de la igualdad social requerida para las sociedades modernas.

El estudio llevado a cabo en Chile por Gubbins (2014) demuestra que la posición socioeconómica de la familia determina notablemente su capacidad de elección de centro, y que, además, esta decisión se ve influida por el deseo de perpetuación dentro de un grupo social preponderante donde el prestigio y el mantenimiento 
de la posición social aporta un capital social a cada familia, es decir, «la necesidad de pertenencia a grupos sociales percibidos con más estatus y cuya integración favorece que los hijos se mantengan en la élite social y económica del país» (p. 1084). Por lo tanto, las familias que social y económicamente puede permitirse matricular a sus hijos en escuelas con un nivel socioeconómico más elevado participarán en la conservación del estatus y la diferenciación de clases muy en consonancia con lo que ya decía Burnar (2010).

Si prestamos atención a la libertad de elección como una expresión percibida desde la óptica del mercado parece destacar la idea de Da Silveira (2012) que percibe el proceso a través de una doble cara, la libertad de oferta y la libertad de demanda, o lo que es lo mismo, la libertad de creación de centros y la libertad de elección de escuela. Lo que está claro es que «para que alguien pueda elegir el tipo de educación que prefiere para sus hijos es necesario que exista una diversidad de opciones» (Da Silveira, 2012, p. 20). Esto genera competencia entre centros por el logro del mayor número de alumnos posibles. Los defensores de este modelo ven en la competencia y su regulación una vía de mejora en la calidad educativa e incluso en la equidad (Prieto y Villamor, 2012), que derivaría en una mayor autonomía para los centros a fin de poder distinguirse y combatir dentro del mercado. «Se fuerza a las escuelas a competir entre sí, lo que, a su vez, las induciría a desarrollar acciones para mejorar la calidad de su oferta junto con una mayor diversificación de la composición social de su estudiantado» (Gubbins, 2014, p. 1069). Pero, como defiende Levin, et al (2013), las escuelas que atienden a poblaciones con altos niveles de pobreza, clase trabajadora o minorías, muestran unos resultados bastante peores que aquellas que cuentan en su mayoría con estudiantes de clase media-alta.

La libertad de elección, según recoge la literatura, se incrementaría a través de cinco dimensiones expuestas por Elacqua, Martínez, Santos, y Urbina (2012, p. 112):

mayor número de alternativas escolares, más diversidad de los proyectos educativos disponibles, más información pública sobre los resultados educativos de cada establecimiento, menor capacidad de selección de estudiantes por parte de las escuelas y menor uso de criterios de asignación de estudiantes a colegios por su habilidad o lugar de residencia

La libertad de elección, como destacan Villalobos y Quaresma (2015), vendría a ser una expresión de la libertad individual, pero ¿hacia dónde nos lleva?, es decir, en muchos casos, ¿Quién elige?

¿Realmente son los padres los que eligen centro o son los centros lo que eligen a los alumnos? En algunos casos, entre los centros financiados con fondos públicos pero de titularidad privada, sus procesos de admisión pueden ser muy selectivos e impactar frontalmente con la libertad de elección de los padres haciéndolos más inaccesibles (Elacqua et al, 2012).

\section{CONCLUSIONES}

La desigualdad por la libre elección parece demostrada en los estudios analizados en función de la metodología diseñada y definida y que responde al método SLR.

En primer lugar podemos concluir que existen dos visiones claras respecto de la libertad de elección. De un lado los que respaldan la intervención estatal, y del otro, aquellos que prefieren que limite su intromisión dejando margen a los mercados. Los primeros solo ven en la intervención del Estado la manera de procurar la igualdad, mientras que los segundos, confían en la libertad de elección como mecanismos de apertura en la oferta educativa, capaz de llegar a todos los hogares y familias según sus intereses y necesidades.

En segundo lugar, la elección de centro constreñida a la zonificación también puede provocar desigualdad. Las familias ubicadas en zonas marginales se verán abocadas a enviar a sus hijos al centro correspondiente a su área geográfica, probablemente con niveles de calidad peores.

Por último, la desigualdad también nace con las políticas de libre elección. El mercado abre sus puertas a nuevas ofertas educativas pero que en muchas ocasiones, bien sea por razones económicas o incluso de desinformación (Elacqua et al, 2012) generan grandes diferencias entre unos sectores poblacionales y otros. Las familias de clase media-alta tienen mayores opciones para elegir porque pueden acceder a una mayor oferta educativa. Las familias de clase baja ven limitado su radio de actuación y en muchas ocasiones, aunque existan mecanismos que permitan la elección tienen que conformarse con la escuela de su barrio.

En definitiva, las políticas de libre elección, como se desarrolla en la literatura analizada, han aumentado la oferta educativa, pero con ello han acrecentado aún más las desigualdades sociales. 


\section{REFERENCIAS BIBLIOGRÁFICAS}

Bartholo, T. L. (2013). Measuring Between-School Segregation in an Open Enrollment System: The Case of Rio de Janeiro. Journal of School Choice: International Research and Reform, 7 (3), 353-371.

Brando, N. (2017). Between equality and freedom of choice: Educational opportunities for the least advantaged. International Journal of Educational Development, 53, 71-79.

Burnar, N. (2010). The Geographies of Education and Relationships in a Multicultural City: Enrolling in High-Poverty, Low-Performing Urban Schools and Choosing to Stay There. Acta Sociológica, 53 (2), 141-159.

Da Silveira, P. (2012). Libertad de Enseñanza, Libertad Religiosa, Secularización y Laicidad: Límites Confusos y Falsas Asociaciones. Páginas de Educación, 5 (1), 17-35.

Elacqua, G., Martínez, M., Santos, H., y Urbina, D. (2012). Tensiones público-privado y el diseño de los sistemas educativos: ¿Qué nos dice PISA? Pro-Posições, Campinas, 23, (2), 105- 123.

Gough, D., Thomas, J., and Oliver, S. (2012). Clarifying differences between review designs and methods, Systematic Reviews. Recuperado el 22 de abril de 2018 de https://link.springer.com/content/pdf/10.1186\%2F2046-4053-1-28.pdf.

Gubbins, V. (2014). Estrategias educativas de familias de clase alta: un estudio exploratorio. Revista Mexicana de investigación Educativa, 19 (63), 1069-1089.

Levin, H. M., Cornelisz, I., y Hanisch-Cerda, B. (2013). Does educational privatisation promote social justice?. Oxford Review of Education, 39 (4), 514-532.

Mazzoli, L., Todd, L., y Laing, K. (2018). Students' views on fairness in education: the importance of relational justice and stakes fairness. Research Papers in Education, 33 (3), 336-353.

Narodowski, M., Gottau, V., y Moschetti, M. (2016). Quasi-State monopoly of the education system and socio-economic segregation in Argentina. Policy Futures in Education, 14 (6), 687- 700.

Prieto, M., y Villamor, P. (2012). Libertad de elección, competencia y calidad: las políticas educativas de la Comunidad de Madrid. Profesorado Revista de Currículum y Formación del Profesorado, 13 (3), 127-144.

Sánchez-Meca, J. (2010). Como realizar una revisión sistemática y un meta-análisis. Aula Abierta, 38 (2), 53-64.

Savage, G. C. (2012). Being different and the same? The paradoxes of 'tailoring' in education quasi - markets. Journal of Pedagogy, 3 (2), 279-302.

Tabatadze, S., y Gorgadze, N. (2018). School voucher funding system of post-Soviet Georgia: From lack of funding to lack of deliverables. Journal of School Choice, 12 (2), 271-302.

Varjo, J., y Kalalahti, M. (2014). Families, school choice, and democratic iterations on te right to education and freedom of education in finnis municipalities. Journal of School Choice, 48, 20-48.

Verger, A., Fontdevila, C., y Zancajo, A. (2017). The Privatization of Education: a political economy of Global Education Reform. New York: Teachers College Press.

Villalobos, C., y Quaresma, M. L. (2015). Sistema escolar chileno: características y consecuencias de un modelo orientado al mercado. Convergencia Revista de Ciencias Sociales, 69, 63- 84. 